\title{
Are the Triptans for Migraine Therapy Worth the Cost?
}

\author{
W. J. Becker
}

\begin{abstract}
The triptans represent a major advance in migraine therapy but their cost per dose greatly exceeds that of many older treatments. There is evidence that for a significant proportion of migraine patients these new drugs can show a positive cost benefit and also improve quality of life. Cost benefit would be expected to be greatest in patients with more severe migraine attacks.
\end{abstract}

RÉSUMÉ: Les triptans en valent-ils le coût dans le traitement de la migraine? Les triptans représentent un avancement important dans le traitement de la migraine, mais leur coût unitaire dépasse largement celui de plusieurs traitements anciens. Cependant des données montrent que chez une proportion significative de migraineux ces médicaments peuvent avoir un rapport coût/bénéfice positif et améliorer la qualité de vie. On peut s'attendre à ce que le coût/bénéfice soit plus élevé chez les patients qui ont des accès de migraine plus sévères.

Can. J. Neurol. Sci. 2000; 27: 111-115

When the first triptan (sumatriptan) reached the Canadian market in 1992, both patients and physicians were delighted to have a new effective drug for the symptomatic treatment of migraine. Sumatriptan also ushered in a whole new era for migraine therapy in terms of cost per dose. Its high cost shocked many patients and physicians and to this day remains controversial. Table 1 shows the relative costs of some drugs used in migraine treatment, as obtained from pharmacies in Alberta.

Nevertheless for many patients with migraine, sumatriptan also ushered in a whole new era of effectiveness. This review will address the issue of whether triptans are worth the price.

Most of the data presented in this review will deal with subcutaneous sumatriptan, as most of the published work on the pharmacoeconomics of the triptans has been done with this drug preparation. Subcutaneous sumatriptan is a good test case for the triptans, because even though it is the most effective triptan preparation in terms of speed of action and overall efficacy rates, ${ }^{1}$ it is also the most expensive (Table 1 ).

\section{Pharmacoeconomicanalysis}

Several types of pharmacoeconomic analyses are used, depending upon whether direct health care costs alone are considered or whether lost work time or pain and suffering are also included. Brief working definitions are given below for three of the more commonly used analysis types. All three will be touched upon to some extent in this review.

Cost effectiveness: This analysis compares two drug treatments in terms of the direct health care costs of treating a medical condition. ${ }^{2}$

Cost benefit analysis: Here two treatments are compared again, but in addition to direct cost to the health care system, the indirect costs of the illness are also considered, including lost wages or productivity.

Cost utility analysis: In this analysis, quality of life is also considered. This requires subjective assessments. In the end, one can come up with the cost of a symptom free year of life produced by a new treatment over and above the benefit produced by an older treatment (a quality adjusted life year or QUALY).

\section{Cost EFFECTIVENESS}

Several studies have examined whether subcutaneous sumatriptan reduces the direct health care costs of migraine. In a study involving 126 patients, Cohen ${ }^{3}$ compared health care resource utilization in the twelve months before to the six months after subcutaneous sumatriptan was made available to patients in a health maintenance organization (HMO) setting. To be eligible, patients had to have between two and six moderate and/or severe migraine attacks per month. Patients could treat an unlimited number of migraine attacks with sumatriptan provided by the investigator, and were encouraged to use it as the first treatment for migraine attacks. Patients used an average of 4.7 injections/month. Resource use measurements were obtained from HMO medical and pharmacy records. The main findings of

From the Department of Clinical Neurosciences, Faculty of Medicine, University of Calgary, Calgary, Alberta, Canada.

RECEIVEDSEPTEMBER 13, 1999. ACCEPTEDIN FINALFORMJANUAR Y 11, 2000. Reprint requests to: W J Becker, Division of Neurology, 12th Floor, Foothills Medical Centre, 1403 - 29th Street NW, Calgary, Alberta T2N 2T9 Canada. 
Table 1: Approximate drug costs per headache*

\begin{tabular}{ll}
\hline acetaminophen $300 \mathrm{mg}$ with codeine $30 \mathrm{mg}$ (4 tablets) & $\$ 0.90$ \\
cafergot $1 \mathrm{mg}$ ( 2 tablets) & $\$ 1.50$ \\
naproxen $550 \mathrm{mg}$ ( 2 tablets) & $\$ 1.70$ \\
butorphanol $1 \mathrm{mg}$ (2 sprays) & $\$ 8.00$ \\
dihydroergotamine subcut, $1 \mathrm{mg}$ & $\$ 6.30$ \\
dihydroergotamine nasal spray, $2 \mathrm{mg}$ & $\$ 10.60$ \\
sumatriptan nasal spray, $20 \mathrm{mg}$ & $\$ 13.80$ \\
sumatriptan, $50 \mathrm{mg}$ & $\$ 14.40$ \\
naratriptan, $2.5 \mathrm{mg}$ & $\$ 14.40$ \\
zolmitriptan, $2.5 \mathrm{mg}$ & $\$ 14.40$ \\
sumatriptan, $100 \mathrm{mg}$ & $\$ 15.20$ \\
sumatriptan subcut, $6 \mathrm{mg}$ & $\$ 40.00$
\end{tabular}

* Costs are rounded off, include dispensing fee, and may vary from location to location, and with amount prescribed at one time. Several assumptions are made. For example, it is assumed that a patient will require a second dose of acetaminophen with codeine, and also of naproxen, but that one dose of a triptan will be sufficient.

this study were that migraine-related physician visits decreased by $50 \%$ and urgent care visits fell by more than $60 \%$ after sumatriptan was started. These savings were not quantitated in dollar terms, and drug costs were not calculated.

Streator et al, ${ }^{4}$ also in an HMO population, examined whether the advent of injectable sumatriptan reduced patient health care costs. Patient utilization of resources was compared from medical records for a six month period before the availability of subcutaneous sumatriptan to a six month period afterward. To be eligible, patients had to have received at least one sumatriptan prescription during 1994. This study included only 49 patients. For this patient population, the number of physician office visits and emergency room visits did not go down, and pharmacy costs per person per month increased dramatically from $\$ 8.45$ to $\$ 57$.
It was concluded that injectable sumatriptan did not reduce the overall consumption of health care services for the treatment of migraine headache. However, patient numbers in this study were very small, and no details were given as to the type of patient for whom sumatriptan was prescribed.

Legg et $\mathrm{al}^{5}$ evaluated 164 patients who received at least one subcutaneous sumatriptan prescription as identified through HMO pharmacy records in 1994. Through a telephone interview, patients self-reported their typical monthly migraine-related health resource utilization rates before and after sumatriptan treatment. Sumatriptan utilization was determined directly from pharmacy records. The results of this analysis are summarized in Table 2. As can be seen, migraine-specific health care utilization costs decreased in many areas once subcutaneous sumatriptan was started, with an average overall cost saving of $\$ 118$ per patient per month. A major criticism of this study might be that utilization data was obtained primarily by patient recall. The authors cite references that the validity of self-reported data compares favourably with that of data from medical records, but one has to wonder whether patient enthusiasm for sumatriptan may have affected their results.

In summary, the actual cost effectiveness of the triptans needs to be examined further, although there is some evidence in the literature that, at least in certain patient populations, sumatriptan by injection can be very cost effective. The migraine population studied by Legg et $\mathrm{al}^{5}$ likely represented the more severe end of the migraine spectrum, as the authors estimated that only four percent $(4 \%)$ of the migraine sufferers in their HMO received sumatriptan during that time period.

It is in this severe migraine subpopulation where sumatriptan may have its greatest cost effectiveness.

\section{WORK-RELATED DISABILITY DUE TO MIGRAINE}

Migraine causes significant disability in some patients, and this can result in missed days of work, or reduced effectiveness if the patient remains at work with symptoms.

How much migraine headache interferes with work depends upon the population studied. In the population-based study, based on the random digit dialling method, von Korff ${ }^{6}$ found

Table 2: Monthly migraine specific health care utilization and cost before and after subcutaneous sumatriptan treatment $(\mathrm{n}=157)$

\begin{tabular}{|c|c|c|c|c|c|}
\hline \multirow[b]{2}{*}{ Service } & \multirow[b]{2}{*}{ Cost PerUnit (US\$) } & \multicolumn{2}{|c|}{ Before } & \multicolumn{2}{|c|}{ After } \\
\hline & & Monthly use rate & $\begin{array}{l}\text { Average cost } \\
\text { per patient }\end{array}$ & Monthly use rate & $\begin{array}{l}\text { Average cost } \\
\text { per patient }\end{array}$ \\
\hline Hospitalization & 1950 & 0.04 & 79.70 & 0.02 & 29.46 \\
\hline Emergency Dept visit & 200 & 0.36 & 71.34 & 0.13 & 26.75 \\
\hline Physician visits & 58 & 1.05 & 60.77 & 0.58 & 33.80 \\
\hline Other prescriptions & 20 & 2.65 & 52.99 & 1.19 & 23.82 \\
\hline Sumatriptan prescriptions & 60 & 0 & 0 & 0.73 & 43.78 \\
\hline Total cost & & & 286.20 & & 168.13 \\
\hline
\end{tabular}

Modified from Legg RF, et al. Am J Man Care 1997;3:117-122 
that, on average, migraine sufferers missed 0.36 days of work per month because of headache. In addition, because of days worked at reduced efficiency due to headache, migraine sufferers or their employers lost an additional 1.01 work day equivalents/ month.

In total then, in this population-based study, migraine patients lost the equivalent of 1.37 work days/month. However, this lost work time was concentrated in a relatively small subpopulation of the migraine sufferers. Sixty percent $(60 \%)$ of the patients had no missed work days, although the great majority of migraine sufferers did have some work days worked with reduced efficiency because of migraine headache. One of the strengths of this study was that the data was based on patient diary recordings made over a three month period and the patient sample was population-based and quite large - 122 patients who worked three days per week or more.

Cortelli et $\mathrm{al}^{7}$ studied patients who were having between two and six moderate or severe migraine attacks/month, who were taking prescription medications for migraine, and who had had two or more physician visits for migraine in the previous year. The data analysis involved 243 patients who worked full time and who reported that headache had caused them to miss some work or non-work activity. Five patients who worked full-time were excluded because they had not missed any activities. On average, these patients lost one work day per month (assuming an eight hour work day, and including reduced effectiveness on days worked with symptoms). Although this was not a population-based study, these results are quite similar to those of von Korff. $^{6}$ One might have expected this study by Cortelli et $\mathrm{al}^{7}$ to have shown a greater number of work days lost as the exclusion of patients with less than two migraine attacks per month would have excluded many milder migraine sufferers. However, the study by Cortelli et al also excluded migraine sufferers with more than six headaches a month. In addition, although the von Korff study was a population-based study, only $55 \%$ of those invited to participate in the diary study to record lost work agreed to participate, and these had a somewhat higher headache pain intensity than the general population sample from which they were drawn. These factors may explain the similar results in the two studies. Both studies would have excluded migraine patients with migraine so severe they were unable to hold down a job.

Legg et $\mathrm{al}^{8}$ examined a clinical migraine population from an HMO. To be included, patients had to have received a prescription for injectable sumatriptan as identified from pharmacy records during 1994, and had to work full time. This patient population likely represents the more severe end of the migraine spectrum, as it was estimated that it comprised only $4 \%$ of the HMO's migraine population. One would assume that the subcutaneous sumatriptan would have been prescribed at this early stage in its market course for more severe migraine patients only. One hundred and one (101) patients of 164 interviewed for this study were working full time, and formed the basis of this analysis. Again, patients disabled from migraine would have been excluded.

In the study by Legg et al, ${ }^{8}$ on average, patients missed 2.75 work days/month because of headache, and lost another 3.5 work day equivalents because of additional days worked with reduced productivity due to headache symptoms. The total work day equivalents lost per month per patient in this study were 6.25 days/month. This study illustrates that in severely affected migraine subpopulations, work loss can be quite formidable.

The high price which some patients pay in lost work time and productivity due to migraine was confirmed by Fishman et al. ${ }^{9}$ Although published in 1999, the baseline data for this study was gathered in 1989 and 1990, prior to the availability of sumatriptan in the United States. In a patient population drawn from patients who had been diagnosed in an HMO as suffering from migraine, he calculated that for those who were working full time, mean annual reduced productivity costs due to both total and partial disability were $\$ 4,827$ US for men $(n=113)$, and $\$ 6,146$ US for women $(n=164)$. Once again, these productivity losses were much higher for individuals in the top third of the migraine sufferers (graded by severity) as compared to those in the milder thirds.

Not all studies have shown such high headache-related work loss in migraine suffers. Michel, ${ }^{10}$ in a population-based diary study, found that annual sickness-related absenteeism in migraineurs who "worked regularly" was 11.15 days (these were the number of work days missed or interrupted for medical reason). Only 2.18 days per year, however, were related to headache. Considering paid work only, the average indirect cost of headache was $\$ 310$ US per migraineur per year.

\section{Cost Benefit of the Triptans}

Subcutaneous sumatriptan has been shown to reduce lost work time. Cortelli et $\mathrm{al}^{7}$ found that whereas lost workplace productivity was an average of one day/month/patient before sumatriptan, it fell to 0.5 days/month with sumatriptan use.

Likewise, $\operatorname{Legg}^{8}$ found that average workdays missed per month in his population fell from 2.75 days/month to 0.8 days/month after sumatriptan was started. In addition, once sumatriptan was started there was a $21 \%$ reduction in days worked with symptoms, which decreased from an average of six days/month to 4.8 days/month. Patients also had a smaller reduction in productivity on days worked with symptoms. Legg et $\mathrm{al}^{8}$ calculated an average productivity saving of $\$ 435 / \mathrm{month}$, while the cost of sumatriptan to the HMO was only $\$ 43.80 /$ month (patient co-pay was not included).

In a different type of analysis, using diary card data, Davies et $\mathrm{l}^{11}$ showed that oral rizatriptan can also produce a reduction in time lost from work as compared to "usual therapy". In this study, $48 \%$ of patients were using oral sumatriptan as their usual therapy, with the remainder using various analgesics including narcotics, barbiturates and ergotamines. Rizatriptan reduced total work time lost (including both absenteeism and work time lost due to reduced efficiency) per migraine attack occurring during work from 5.3 to 3.0 hours.

\section{TRIPTANS AND QUALITY OF LIFE}

Measuring the cost utility of migraine treatment is complex and involves many subjective judgments. It will not be discussed here, but it is important to point out that a number of studies have shown improved health-related quality of life after sumatriptan therapy was started. Dahlof et al $^{12}$ found that scores on most short form 36 health survey dimensions were significantly higher after three months of sumatriptan therapy. The same was true for quality of life as measured by the migraine-specific quality of 
life questionnaire. There seems little doubt then that the triptans have the potential to reduce migraine pain and suffering over other therapies, at least in some subpopulations of migraine patients. It is difficult, however, to place a dollar value on this benefit.

\section{Clinicalapproach}

For which patients with migraine does sumatriptan show a positive cost effectiveness and/or cost benefit? The triptans clearly would show neither as compared to other therapies in patients with migraine mild enough to respond well to much less expensive medications and whose attacks did not interfere with the patients'activities.

Cost effectiveness can be achieved primarily in patients who are significant utilizers of health care resources such as other medications, physician visits, hospital emergency rooms, etc. In some ways, a strict cost effectiveness analysis which considers only direct health care costs is unfair to the patient, as it puts at a disadvantage those migraine sufferers who largely suffer through their possibly severe, but temporary attacks, with inexpensive but only partially effective medications. It is difficult for the more expensive medications to demonstrate cost savings in such patients unless work time lost is taken into account, or some value is placed on pain and suffering. It has been estimated that $76-86 \%$ of the total cost of migraine is due to lost labour costs, with only $14-24 \%$ a result of direct medical expenditures. ${ }^{13}$ Furthermore, the direct costs of migraine can be easily underestimated if one does not take into account the large amount of money spent by migraine patients on "alternative therapies". Van Roijen ${ }^{14}$ found, for the year 1988 (pretriptan era), that whereas drug costs for migraine sufferers were only 11.5 million NLG, alternative practitioner costs were 106.7 NLG. The lost productivity costs related to migraine were, however, estimated at over 500 million NLG, considering both absence from work and reduced productivity while at work.

It seems clear that to be cost effective, sumatriptan should be prescribed primarily for migraine sufferers who do not respond adequately to significantly less expensive medications. Only the individual patient can tell us how well he or she responds to analgesics, nonsteroidal anti-inflammatory drugs (NSAID) and ergotamine. Another consideration here is the side effect profile of the alternative drugs, and their safety. For example, in contrast to the older ergotamines, including dihydroergotamine, which activate many receptor-types, the triptans are much more specific. This specificity for only a few 5HT receptor subtypes gives the triptans the potential for a more favourable side effect and safety profile and allows the administration of doses adequate to achieve a therapeutic effect.

The triptans are not necessary for every migraine patient. Two direct comparison studies had been done between oral sumatriptan and aspirin plus metoclopramide. One ${ }^{15}$ showed the two treatments to be comparable. Another ${ }^{16}$ found sumatriptan to be superior. In this second study, $70 \%$ of sumatriptan-treated patients reported that they would be prepared to take the study treatment again, compared to $46 \%$ of aspirin/metoclopramidetreated patients. A relatively small study ${ }^{17}$ was unable to show a difference between an NSAID (tolfenamic acid) and oral sumatriptan (but with a total of 154 patients in this placebo- controlled, parallel-group study, its power to detect a difference would be small).

The ergotamines have generally fared less well in comparison trials. In one study, ${ }^{18} 100 \mathrm{mg}$ of sumatriptan was superior to $2 \mathrm{mg}$ of ergotamine (Cafergot). At two hours, 35\% of patients were pain free in the sumatriptan group, compared to only $13 \%$ in the Cafergot group. In another, ${ }^{19}$ eletriptan was significantly more effective than ergotamine. This study, a large placebo-controlled study, highlighted the poor response of migraine patients to ergotamines. For example, at two hours, only $10 \%$ of ergotamine-treated patients were pain free, as compared to $38 \%$ for eletriptan and $5 \%$ for placebo. Side effects were also an issue. For example, whereas $62 \%$ of patients treated with eletriptan were free of nausea at two hours, only $36 \%$ of Cafergot-treated patients were nausea free, compared to $47 \%$ of those who received placebo. In other words, with respect to nausea, Cafergot under-performed placebo.

In a large, placebo-controlled crossover study, ${ }^{20}$ diclofenacpotassium was found to equal sumatriptan $(100 \mathrm{mg}$ ) in a migraine population with an attack frequency of two to six attacks per month.

For some patients, it would appear that less expensive medications work as well as the triptans. The most reasonable approach therefore, would be to tailor the patient's medications to the migraine attack severity. This has been recommended before $^{21}$ and seems the most logical approach. Milder attacks are treated with less expensive drugs such as aspirin, acetaminophen, and ibuprofen. Attacks of moderate severity may respond to the NSAIDs, but some may require triptans. Less expensive combination analgesics can also be used if the patient responds well to these without significant sedation or other side effects, but with a caveat that if the patient has frequent attacks, the need to use large doses of these less effective agents may lead to a chronic daily headache syndrome. ${ }^{22}$ For many patients with severe attacks, as judged by disability, the triptans are often the best choice, although some might still respond sufficiently to NSAIDs or analgesics.

One approach to selecting patients who will benefit most from triptan use is to determine the extent of migraine-related disability over a period of several months by means of a questionnaire. ${ }^{23}$ With this method, a subpopulation of patients, who are unable to function well over a significant number of days per month because of migraine, can be identified. The triptans would be expected to have their greatest cost benefit and/or cost utility in this migraine subpopulation.

Most studies dealing with triptan cost effectiveness and cost utility deal with subcutaneous sumatriptan. More studies using the oral triptans are needed. As the oral triptans are also highly effective drugs for migraine ${ }^{1,24}$ and because their cost is lower as compared to subcutaneous sumatriptan, one would expect them to show a cost benefit in patients with moderate and severe migraine not sufficiently responsive to other less expensive medications.

\section{CONCLUSION}

For many patients with moderate and severe migraine attacks, the triptans, despite their expense, can be cost effective in some and show a cost benefit and cost utility in many. Patient selection 
based on migraine attack severity and experience with other less expensive treatments is essential. However, for many patients with severe attacks, it would appear inappropriate to perform exhaustive trials of less expensive or more hazardous drugs, as they are unlikely to be effective or tolerated. Cost benefit can be enhanced by providing patients with several medication options for symptomatic migraine treatment, as most patients with severe attacks also have some milder attacks. In this way, less expensive options can be utilized for milder attacks.

\section{REFERENCES}

1. Tfelt-Hansen P. Efficacy and adverse events of subcutaneous, oral and intranasal sumatriptan used for migraine treatment: a systematic review based on number needed to treat. Cephalalgia 1998 Oct;18(8):532-538.

2. Shear NH, Oh PI, Arikian SR, Detsky AS, Laupacis A. Pharmacoeconomics: measure of cost and benefit. Ann R Coll Phys Surg Canada 1995;28:239-240.

3. Cohen JA, Beall DG, Miller DW, et al. Subcutaneous sumatriptan for the treatment of migraine: humanistic, economic, and clinical consequences. Fam Med 1996 Mar;28(3):171-177.

4. Streator SE, Shearer SW. Pharmacoeconomic impact of injectable sumatriptan on migraine associated healthcare costs. Am J Manag Care 1996;2:67-76.

5. Legg RF, Sclar DA, Nemec NL, Tarnai J, Mackowiak JI. Cost effectiveness of sumatriptan in a managed care population. Am J Manag Care 1997;3:117-122.

6. von Korff M, Stewart WF, Simon DJ, Lipton RB. Migraine and reduced work performance: a population-based diary study. Neurology 1998;50:1741-1745.

7. Cortelli $\mathrm{P}$, Cahlof $\mathrm{C}$ Bouchard $\mathrm{J}$, et al. A multinational investigation of the impact of subcutaneous sumatriptan III. Workplace productivity and non-workplace activity. PharmacoEconomics 1997;11(Suppl.1):35-42.

8. Legg RF, Sclar DA, Nemec NL, Tarnai J, Mackowiak JI. Cost benefit of sumatriptan to an employer. JOEM 1997;39(7):652657.

9. Fishman P, Black L. Indirect costs of migraine in a managed care population. Cephalalgia 1999;19:50-57.

10. Michel P, Dartigues JF, Duru G, et al. Incremental absenteeism due to headaches in migraine: results from the Mig-Access French national cohort. Cephalalgia 1999;19(5):503-510.

11. Davies GM, Santanello N, Gerth W, Lerner D, Block GA. Validation of a migraine work and productivity loss questionnaire for use in migraine studies. Cephalalgia 1999;19(5): 497-502.

12. Dahlof C, Bouchard J, Cortelli P, et al. A multi-national investigation of the impact of subcutaneous sumatriptan II: health-related quality of life. PharmacoEconomics 1997; 11(Suppl.1):24-34.

13. Osterhaus JT, Gutterman DL, Plachetka JR. Healthcare resource and lost labor costs of migraine in the US. PharmacoEconomics 1992;2:67-76.

14. Van Roijen L, Essink-Bot M-L, Koopmanschap MA, et al. Societal perspective on the burden of migraine in the Netherlands. PharmacoEconomics 1995;7:170-179.

15. Tfelt-Hansen P, Henry P, Mulder LJ, et al. The effectiveness of combined oral lysine acetylsalicylate and metoclopramide compared with oral sumatriptan for migraine. Lancet 1995;346:923-926.

16. The Oral Sumatriptan and Aspirin plus Metoclopramide Comparative Study Group. A study to compare oral sumatriptan with oral aspirin plus oral metoclopramide in the acute treatment of migraine. Eur Neurol 1992;32:177-184.

17. Myllylä VV, Havanka H, Herrala L, et al. Tolfenamic acid rapid release versus sumatriptan in the acute treatment of migraine: comparable effect in a double-blind, randomized, controlled, parallel-group study. Headache 1998;38:201-207.

18. The Multinational Oral Sumatriptan and Cafergot Comparative Study Group. A randomized, double-blind comparison of sumatriptan and cafergot in the acute treatment of migraine. Eur Neurol 1991;31:314-322.

19. Reches A. Comparison of the efficacy, safety and tolerability of oral eletriptan and cafergot for the acute treatment of migraine. Cephalalgia 1999;19:355.

20. The Diclofenac-K/Sumatriptan Migraine Study Group. Acute treatment of migraine attacks: efficacy and safety of a nonsteroidal anti-inflammatory drug, diclofenac-potassium, in comparison to oral sumatriptan and placebo. Cephalalgia 1999;19(4):232-240.

21. Pryse-Phillips WEM, Dodick WD, Edmeads JG, et al. Guidelines for the diagnosis and management of migraine in clinical practice. Can Med Assoc J 1997;156:1273-1287.

22. Mathews NT. Drug induced headache. Neurologic Clinics 1990;8:903-912.

23. Stewart WF, Lipton RB, Whyte J, et al. An international study to assess reliability of the Migraine Disability Assessment (MIDAS) score. Neurology 1999;53(5):988-994.

24. Tfelt-Hansen $\mathrm{P}$, Teal $\mathrm{J}$, Rodriguez $\mathrm{F}$, et al on behalf of the Rizatriptan 030 Study Group. Oral rizatriptan versus oral sumatriptan: a direct comparative study in the acute treatment of migraine. Headache 1998;38:748-755. 